\title{
Cytapheresis as a Non-Pharmacological Therapy for Inflammatory Bowel Disease
}

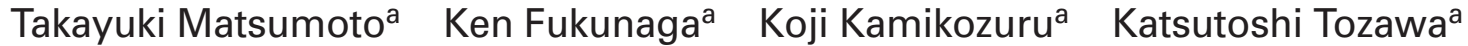 \\ Yoko Yokoyama $^{\mathrm{a}}$ Takeshi Kusaka $^{\mathrm{a}}$ Kunio Onishia Hiroto Miwa ${ }^{\mathrm{b}}$ Shiro Nakamura ${ }^{\mathrm{a}}$ \\ a Division of Lower GI Disease, \\ ${ }^{b}$ Division of Upper GI Disease, Department of Internal Medicine, Hyogo College of Medicine, Nishinomiya, Japan
}

\section{Key Words}

Inflammatory bowel disease - Ulcerative colitis .

Apheresis · Leukocytapheresis · Granulocytapheresis

\section{Summary}

Although inflammatory bowel disease (IBD), including ulcerative colitis (UC) and Crohn's disease (CD), is a chronic recurrent disease with unknown etiology. Recent immunological studies suggest close relation to autoimmune status featured by antibodies against colonic epithelial cells. For patients with IBD, 5-aminosalycilates are often used in case of mild disease, and corticosteroids are standard therapy for moderate-to-severe disease. However, we often encounter patients who are resistant to or dependent of conventional therapy, which are likely to lead to future problems in quality of life due to adverse effects of drugs used, especially corticosteroids. Extracorporeal leukocyte removal therapy (cytapheresis) is one of the adjunctive therapies for IBD patients refractory to steroids. By removing circulating activated leukocytes, especially granulocytes and lymphocytes, impaired immune response is suppressed. In the present article recently published studies are reviewed in order to reflect the current state of the art in the use of cytapheresis for treating IBD, especially UC and CD. Although there are only few randomized controlled trials, clinical experience so far suggests that cytapheresis has superior efficiency than conventional therapies in steroid-resistant moderate-to-severe UC. Moreover, cytapheresis features its safety characteristic compared with other conventional medications for severe UC, cytapheresis is regarded as safe treatment regimen.

\section{Schlüsselwörter}

Inflammatorische Darmerkrankung - Colitis ulcerosa .

Apherese · Leukozytapherese · Granulozytapherese

\section{Zusammenfassung}

Entzündliche Darmerkrankungen wie Colitis ulcerosa und Morbus Crohn sind chronische wiederkehrende Erkrankungen mit unklarer Ätiologie. Neue immunologische Untersuchungen lassen einen durch Antikörper gegen Kolonepithelzellen vermittelten Autoimmunstatus vermuten. Patienten mit leichter inflammatorischer Darmerkrankung werden häufig mit Aminosalicylaten behandelt; Kortikoide sind die Standardtherapie bei mittlerer bis schwerer Erkrankung. Non-Responder dieser Therapieformen sind erheblich in ihrer Lebensqualität eingeschränkt. Die extrakorporale Leukozytenbehandlung durch Zytapherese ist eine Therapieoption für Patienten mit inflammatorischer Darmerkrankung, die steroidrefraktär sind. Durch Zytapherese werden zirkulierende aktivierte Leukozyten, insbesondere Granulozyten und Lymphozyten, entfernt, was mit einer Unterdrückung der Immunantwort einhergeht. Die vorliegende Übersichtsarbeit beschreibt die neuesten Studien zum aktuellen Status der therapeutischen Apherese bei Patienten mit inflammatorischer Darmerkrankung, insbesondere Colitis ulcerosa und Morbus Crohn. Obwohl die Anzahl der randomisierten kontrollierten Studien gering ist, zeigt die bisherige klinische Erfahrung, dass die therapeutische Zytapherese als nichtpharmakologische, immunmodulative Therapie bei steroidrefraktärer mittlerer bis schwerer Colitis ulcerosa effektiv ist. Im Vergleich zur konventionellen Pharmakotherapie bei schwerer Colitis ulcerosa stellt die Zytapherese ein sicheres Behandlungsregime dar.

\begin{tabular}{ll}
\hline KARGER & $\oplus$ 2008 S. Karger GmbH, Freiburg \\
Fax +49 761 45207 14 & Accessible online at: \\
$\begin{array}{l}\text { E-mail Information@Karger.de } \\
\text { www.karger.com }\end{array}$ & www.karger.com/tmh
\end{tabular}




\section{Introduction}

Ulcerative colitis (UC) and Crohn's disease (CD) are chronic intestinal inflammatory diseases of yet unknown origin, which may lead to disabled quality of life (QOL) through long-lasting symptoms such as diarrhea, bloody stool, and abdominal pain [1]. They are subsumed by the term idiopathic inflammatory bowel disease (IBD). In Japan, the number of patients with UC and CD has been increased by a factor of 10 since the 1980s. The etiology of IBD however is unclear. An autoimmune disturbance is thought to play an important role in this incurable disease.

Currently, systemic administration of corticosteroids is the gold standard in the therapy of moderately-to-severe UC, but is likely to cause dose-dependent adverse effects such as moon face, infections, diabetic disease, and osteoporosis. In such steroid-resistant or steroid-dependent patients, immunomodulators such as cyclosporine, tacrolimus, 6-MP, or azathiopurine have been widely used.

Cytapheresis therapy which removes leukocytes from peripheral blood has first been applied in the treatment of UC in 1995 [2]. The primary aim of cytapheresis is to suppress and reduce impaired immune responses in the diseased intestine by removing circulating activated leukocytes, especially granulocytes, which have been shown to cause intestinal crypt abscess. Currently available cytapheresis techniques for active IBD patients are filtration leukocytapheresis (LCA), adsorption granulocyte/monocyte apheresis (GMA), and centrifugal lymphocytapheresis (CLA). GMA and LCA have been approved by the Japanese national health insurance policy for treating active UC since 2000 and 2001, respectively, and have been widely used as non-pharmacological and non-surgical therapeutic option for intractable UC patients. On the other hand, cytapheresis, although GMA is in the final stage for getting the government approval, has never been approved for CD in Japan.

There are only a few data addressing which patients are more likely to respond to cytapheresis therapy and on which physiological mechanism the curative effect of cytapheresis in IBD is based.

\section{Cytapheresis Techniques Currently Available for Patients with Active Inflammatory Bowel Disease}

If cytapheresis techniques were applied in steroid-resistant and/or steroid-dependent patients with active UC, mainly LCA and GMA, but only rarely CLA, are used. In the following, the standard procedures of these techniques are summarized.

\section{Filtration Leukocytapheresis}

LCA is carried out using a Cellsorba $\mathrm{EX}^{\mathrm{TM}}$ (Asahi Kasei Kuraray Medical Co., Ltd., Tokyo, Japan) column which is filled with polyester unwoven filter. This polyester unwoven leukocyte removal filter installed in polycarbonate outer shell is designed to remove almost $100 \%$ of granulocytes and monocytes and $64 \%$ of lymphocytes [3]. Furthermore, approximately $35 \%$ of platelets can be trapped to the filter from processed peripheral blood [3]. LCA is usually performed weekly using a Cellsorba EX set in a simple one-way hemofiltration circuit [2-4]. A roller pump drained peripheral blood of the patient from an antecubital vein under constant flow rate of 30-50 $\mathrm{ml} / \mathrm{min}$. The anticoagulant nafamostat mesilate (Futhan ${ }^{\circledR}$; Torii Pharmacol., Co, Ltd., Tokyo, Japan) or heparin was mixed with saline and was added to the patients' drained peripheral whole blood as anticoagulant before infusion into the column. Although use of nafamostat mesilate is sometimes associated with allergic adverse effects, the half-life of the drug is too short to affect bleeding accompanying ulcerative lesions in UC. Anti-nafamostat mesilate IgE was present in $12 \%$ of the symptomatic UC patients whose adverse effects were highly suspected to be caused by nafamostat [5]. However, in another $43 \%$ of patients anti-nafamostat mesilate IgE could not be detected in spite of the fact that their adverse effects were also highly suspected to be induced by nafamostat.

If the patient is in severely active condition, two sessions are allowed in the first week of the treatment followed by 4 consecutive weekly sessions.

\section{Adsorption Granulocyte/Monocyte Apheresis}

GMA is carried out using the Adacolumn (JIMRO Co. Ltd., Takasaki, Japan) which is filled with cellulose acetate beads. The beads are designed to adsorb about $65 \%$ of granulocytes, $55 \%$ monocytes/macrophages and a smaller fraction of lymphocytes from peripheral blood. One of the mechanisms which adsorb leukocytes to the beads is through so-called Fc-R and complement receptors [6-8]. The circuit diagram for GMA is almost the same as that of LCA. The duration of one GMA session is $60 \mathrm{~min}$, at $30 \mathrm{ml} / \mathrm{min}$ with optimal amount of nafamostat mesilate or heparin as an anticoagulant. As GMA does not remove platelets, this method is preferably used in patients with moderate-to-severe bleeding.

\section{Centrifugal Lymphocytapheresis}

CLA is performed using a centrifugal cell-separator (Component Correction System: CCS, Haemonetics Japan, Tokyo, Japan). Peripheral whole blood drained from patient is collected into a polycarbonate disposable bowl. CCS gives the $125 \mathrm{ml}$ bowl spin to generate centrifugal force, and the refined 
lymphocyte-rich layer (buffy coat) is then removed selectively. After the lymphocyte removal, the separated blood is re-transfused to the patient via the same catheter. CLA processes approximately $2,400 \mathrm{ml}$ whole blood. In LCA for patients with $\mathrm{UC}$, the relatively heavy layer of the leukocytes is removed to increase efficacy.

\section{Cytapheresis for Patients with Ulcerative Colitis}

LCA and GMA have been widely used in Japan as an effective therapeutic option for patients with active UC; however, our current level of knowledge for this unique therapy is still fragmentary and based on empiric recommendations.

\section{LCA for UC}

Although the mechanisms underlying the therapeutic efficacy of LCA treatment have not been fully elucidated, immune modulations such as cytokine production and immune regulation induced during LCA were reported previously. It was demonstrated that LCA enhances the ability of the peripheral lymphocytes to produce the anti-inflammatory cytokine IL-4 [12]. Furthermore, LCA was shown to decrease IL-6 release (a pro-inflammatory cytokine) from peripheral blood lymphocytes, accompanying a concomitant increase of IL-10 production. These modifications in inflammatory and anti-inflammatory cytokine production may induce inhibition of IL-1 at the protein and mRNA expression level during the cytapheresis procedure [13].

On the other hand, approximately $35 \%$ of peripheral blood platelets adhere onto the surface of polyester filter of Cellsorba and thus are removed by LCA [4]. Recently, it has been reported that circulating platelets are important cells not only in hemostasis, but also in a variety of inflammatory responses [14]. An increase of peripheral platelet count has often been recognized as a common feature during chronically active IBD [15]. As reported previously, the high platelet number correlates well with disease severity [16]. We therefore hypothesized that the significant platelet removal achieved during LCA might play an active role in down-regulating severe immunological reactions in UC patients with an acute flare. We have proven that the clinical efficacy of LCA in severe UC patients can be predicted by the reduction of activated platelets achieved during the first LCA session [17].

Sawada et al. [3] conducted a randomized multicenter trial on the effects of LCA in patients with active UC. UC patients who were resistant to conventional steroid therapy with at least $30 \mathrm{mg}$ /day were randomized to either receive LCA (adding LCA without increasing the corticosteroid dose) or high-dose steroid therapy (h-PSL; increasing the corticosteroid dose to $60 \mathrm{mg} /$ day). LCA showed a significantly higher efficacy compared with conventional h-PSL (74.1 vs. $31.8 \%$; $\mathrm{p}<0.05)$. However, no significant difference in clinical efficiency between LCA and h-PSL was found in steroid-naïve UC patients. The major advantage of LCA was the better safety profile, showing no serious adverse side effect, while a substantial number of patients in the h-PSL group suffered from severe adverse effects such as infection.

\section{GMA for $U C$}

Several clinical studies [18-22] showed remission induction by GMA in patients with UC. As stated above, GMA utilizes cellulose acetate beads, which adhere granulocytes through Fc receptors. Cellular contact to the beads may exert other immunological effects to other types of cells such as lymphocytes. We have demonstrated that at the first session of GMA, the proportion of regulatory $\mathrm{T}$ cells $\left(\mathrm{CD} 25^{\text {high }} \mathrm{CD} 4+\mathrm{T}\right.$ cells) in the peripheral blood of patients with UC increases; these cells may suppress impaired immune responses in UC. In patients with active IBD, peripheral blood granulocytes and monocytes/macrophages are elevated and show activation behavior and increased survival time [23-28]. As these leukocytes are the major sources of inflammatory cytokines [29,30], they may contribute to the exacerbation and perpetuation of IBD [31, 32]. Furthermore, the level of neutrophil infiltration into the mucosal tissue in patients with active IBD is related to the severity of intestinal inflammation and clinical relapse [33-35].

We showed that peripheral regulatory $\mathrm{T}$ cell expression, which is suppressed in active UC, was significantly increased after a single GMA session [36]. Impaired activity and/or proportion of regulatory $\mathrm{T}$ cells results in over-activation of immune responses, including polyclonal antibody production, and leads to autoimmunity-mediated tissue destruction. The increase in CD25 $5^{\text {high }} \mathrm{CD} 4+$ regulatory $\mathrm{T}$ cells after GMA should contribute to improved immune function of the patient. This rise could reflect depletion of non-CD25 $5^{\text {high }} \mathrm{CD} 4+\mathrm{T}$ cells. Likewise, several other investigators reported favorable immunological observations associated with GMA [37, 38]. Andoh et al. [38] reported a significant decrease of IL-1 $\beta$ - and TNF- $\alpha$ induced IL- 8 and IL-6 release from peripheral leukocytes following GMA. In conclusion, GMA may correct a part of impaired immune responses through regulatory $\mathrm{T}$ cells and cytokine production from lymphocytes in the peripheral blood. The mechanisms how the changes in peripheral blood immune cells cause changes in intestinal immune cells should be further examined. A multicenter trial of GMA for active UC patients [39] showed that GMA had a significantly higher efficiency for relapsing UC patients than conventional h-PSL therapy (GMA vs. h-PSL $=54.8$ vs. $39.5 \%$; $p<0.05$ ), and GMA had a significantly lower ratio of adverse events compared with h-PSL (GMA vs. h-PSL $=89.9$ vs. $58.9 \%$; $\mathrm{p}<0.001)$. 


\section{Cytapheresis for Patients with Crohn's Disease}

In order to learn more about the efficacy of cytapheresis in patients with active $\mathrm{CD}$, we have aimed to conduct a multicenter open label study on GMA in Japan for patients with active CD refractory to more than $1,200 \mathrm{kcal} /$ day enteral nutrition with elemental diet [9]. Prior to this multicenter study, we originally checked the efficacy of cytapheresis in active CD in a preliminary clinical trial [10]. According to these two studies, GMA showed preferable effects in patients with active CD with colonic disease as a major involvement. As shown by Fukuda et al. [7], significant improvements in CDAI, IOIBD, and IBDQ scores were observed at week 7 of GMA therapy. Before CMA, the mean values of the CDAI, IOIBD and IBDQ score were 275.6, 3.4, and 152, respectively. The corresponding values after GMA were $214.8(\mathrm{p}=0.0005), 2.54$ ( $\mathrm{p}=0.0224)$, and $165(\mathrm{p}=0.0327)$. Currently, GMA for CD is considered as an option of medical therapy, and it is on the final stage for getting the government approval. Although LCA has been reported to have superior efficiency in patients with active CD [11], there is no sufficient data eligible for getting approval from the government.

\section{Cytapheresis for Inflammatory Bowel Disease in the USA}

In the USA, S.B. Hanauer (The University of Chicago) and L.F. Mayer (Mount Sinai School of Medicine) have been conducted a pilot study of LCA for patients with active UC refractory to steroid and 5-aminosalylic acid (5-ASA). However, their treatment schedule and column were different from those of the Japanese trial. Their regimen was designed to perform LCA twice a week for 3 weeks (in total 6 sessions), and the column (Cellsorba FX) used in the study was modified to be usable under acid-citrate dextrose sodium (ACD) instead of nafamostat mesilate or heparin as an anticoagulant. In total, 12 steroid-refractory and 24 5-ASA-refractory UC patients have been set as their goal for this pilot study.

In the USA, GMA has also been evaluated for patients with moderate-to-severe active UC in a multicenter double blind, randomized, sham-controlled trial. This trial has been also conducted by Hanauer et al. and scheduled to perform GMA twice a week for 3 weeks as in the US trial for LCA. The number of the patients enrolled currently is 168 cases (September 2006).

\section{Cytapheresis for Inflammatory Bowel Disease in Europe}

LCA got a CE mark and has been approved as medical device in Europe. The column used in Europe is Cellsorba FX which is modified to use with ACD as in the USA. A clinical trial has been started for active UC refractory to conventional medica- tion therapy in Germany. I. Emmrich (University of Rostock) has been conducted the trial which consisted of two phases. In the first phase, patients have been enrolled to 5 weekly GMA series. The responder, who could be induced to their clinical remission after the first phase, has been enrolled into the second phase, a randomized controlled trial. Patients enrolled to the second phase have been randomly divided into two groups of either continuing 5 monthly GMA sessions or receiving conventional medication alone. The number of the patients enrolled currently is total 20 (September 2006). Moreover, in Israel, Italy, Spain, Sweden, and the UK other clinical trials have been carried out.

A multicenter randomized, double blind, sham-controlled trial of GMA for patients with acute UC has been carried out in EU countries of Austria, Belgium, Denmark, France, Germany, Italy, Norway, Spain, Sweden, and the UK. The protocol in this European trial has been designed to be same as in the US trial mentioned above. The total number patients enrolled currently is 51 (September 2006).

\section{Future Development in Cytapheresis for the Management of Inflammatory Bowel Disease}

Factors which may influence clinical effectiveness of LCA and GMA include blood flow speed $(\mathrm{Qb})$, proceeding time, and proceeding frequency (Qf). Basically, slower Qb increase the leukocyte removal rate of the column. However, with Cellsorba, the column used in LCA, coagulation problems in the column may arise at Qb lower than $20 \mathrm{ml} / \mathrm{min}$ since the platelets in the column cause foamy thromboses. On the other hand, Adacolumn, the column used in GMA, adsorbs granulocytes, monocytes/macrophages and a smaller fraction of lymphocytes from patient's peripheral whole blood on cellulose acetate beads filled with the device. As peripheral blood leukocytes bear a Fc R and complement receptors $[8,40]$ and the column is not likely to adhere platelets, GMA is suitable for processing under slow $\mathrm{Qb}$ conditions. We, then, focused on platelet removal performances of LCA and GMA as a possible factor to understand their therapeutic mechanisms. GMA may be better if the patient is in severe inflammatory condition and/or dehydrated status because of blood hyperviscosity. GMA is also recommended for patients bearing high risks such as younger age, older age, and small body weight. Conversely, LCA can be primarily used for patient with severe inflammatory condition of the colonic mucosa, such as grade 4 in the Matts' classification [41] which is often seen in intractable patients. We hypothesized that the platelet reduction achieved during LCA might amplify its therapeutic efficiency for UC patients with severe mucosal damage by restoring microblood circulation in the colonic mucosa.

The clinical response to cytapheresis (clinical and endoscopic indices) is usually seen at week 5. During this period, there could be serious deteriorations together with debilitating im- 
pact on the QOL for patients who become non-responder. Since established evidence suggest that both functional suppression of the circulating leukocytes and the quantitative removal of activated leukocytes contribute to the efficacy of this non-pharmacological therapy, it has been hypothesized that there might be an inverse proportion between Qf and immunological effect of cytapheresis. A recent unpublished randomized controlled study comparing intensive and once a week regimen showed that intensive treatment, which consists of two aphereses in a week for 5 consecutive weeks, results in higher remission rates according to both clinical and endoscopic criteria and shorter duration before getting clinical response.

Although, for steroid-resistant severe UC, intravenous infusion therapy of cyclosporine has been widely accepted as an adjunct therapy [42], a large proportion of patients may have surgery in future years. Clinical experience so far suggests that cytapheresis has superior efficiency as a non-pharmacological immunomodulative therapy for steroid-resistant UC patients before colectomy [1-4].

\section{Conclusion}

Cytapheresis is an effective non-pharmacological therapy for steroid-resistant moderate-to-severe UC. We have to clarify which patients are good candidates for cytapheresis and what are the mechanisms of action in UC. Clinical efficacy and indication based on disease activity for patients with steroid-naïve UC should be determined to improve QOL of the patients.

\section{References}

1 Hibi T, Ogata H: Novel pathophysiological concepts of inflammatory bowel disease. J Gastroenterol. 2006;41(1):10-6.

2 Sawada K, Ohnishi K, Fukui S, et al: Leukocytapheresis therapy, performed with leukocyte removal filter, for inflammatory bowel disease. J Gastroenterol 1995;30:322-9.

3 Sawada K, Muto T, Shimoyama T, et al: Multicenter randomized controlled trial for the treatment of ulcerative colitis with a leukocytapheresis column. Curr Pharmaceutical Design 2003;9:307-21.

4 Shibata H, Kuriyama T, Yamawaki N: Cellsorba. Ther Apher Dial 2003;7:44-7.

$\checkmark 5$ Nagase K Fukunaga K, Ohnishi K, Kusaka T, Matoba Y, Sawada K: Detection of specific IgE antibodies to nafamostat mesilate as an indication of possible adverse effects of leukocytapheresis using nafamostat mesilate as anticoagulant. Ther Apher Dial 2004;8(1):45-51.

6 Saniabadi AR, Hanai H, Suzuki Y, et al: Adacolumn for selective leukocytapheresis as a non-pharmacological treatment for patients with disorders of the immune system: an adjunct or an alternative to drug therapy? J Clin Apher 2005;20:171-84.

7 Hiraishi K, Takeda Y, Shiobara N, et al: Studies on the mechanisms of leukocyte adhesion to cellulose acetate beads: an in vitro model to assess the efficacy of cellulose acetate carrier-based granulocyte and monocyte adsorptive apheresis. Ther Apher Dial 2003;7:334-40.

8 D'Arrigo C, Candal-Couto JJ, Greer M, et al: Human neutrophil Fc receptor-mediated adhesion under flow: a hallow fiber model of intravascular arrest. Clin Exp. Immunol 1993;100:173-9.

-9 Fukuda Y, Matsui T, Suzuki Y, et al : Adsorptive granulocyte and monocyte apheresis for refractory Crohn's disease: an open multicenter prospective study. J Gastroenterol 2004;39:1158-64.

10 Kusaka T, Fukunaga K, Ohnishi K, et al: Adsorptive monocyte-granulocytapheresis (M-GCAP) for refractory Crohn's disease. J Clin Apher 2004;19: 168-173.

11 Kosaka T, Sawada K, Ohnishi K, et al: Effect of leukocytapheresis therapy using a leukocyte removal filter in Crohn's disease. Intern Med 1999; 38:102-11.
12 Noguchi M, Hiwatashi N, Hayakawa T, Toyota T: Leukocyte removal filter-passed lymphocytes produce large amounts of interleukin-4 in immunotherapy for inflammatory bowel disease: Role of bystander suppression. Ther Apher 1998;2: 109-14.

13 Hanai H, Iida T, Takeuchi K, et al: Decrease of reactive-oxygen -producing granulocytes and release of IL-10 into the peripheral blood following leukocytapheresis in patients with active ulcerative colitis. World J Gastroenterol 2005;11:3085-90.

14 Danese S, del la Motte C, Fiocchi C: Platelets in inflammatory bowel disease: clinical, pathogenic, and therapeutic implications. Am J Gastroenterol 2004; 99(5):938-45.

15 Talstad I, Rootwelt K, Gjone E: Thrombocytosis in ulcerative colitis and Crohn's disease. Scand J Gastroenterol 1973;8:135-8.

16 Harries AD, Fitzsimons E, Fifield R, Dew MJ, Rhoades J: Platelet count: a simple measure of activity in Crohn's disease. Br Med J 1983;286:1476.

17 Fukunaga K, Fukuda Y, Yokoyama Y, et al: Activated platelet as a possible early marker to predict clinical efficacy of leukocytapheresis in severe ulcerative colitis patients. J Gastroenterol 2006;41: 524-32.

18 Kanke K, Nakano M, Hiraishi H, et al: Evaluation of granulocyte/monocyte apheresis therapy for active ulcerative colitis. Dig Liv Dis 2004;36:512-8.

19 Naganuma M, Funakoshi S, Sakuraba A, et al: Granulocytapheresis is useful as an alternative therapy in patients with steroid-refractory or -dependent ulcerative colitis. Inflamm Bowel Dis 2004; 10:251-7.

20 Yamamoto T, Umegae S, Kitagawa T, et al: Granulocyte and monocyte adsorptive apheresis in the treatment of active distal ulcerative colitis: a prospective, pilot study. Aliment Pharmacol Ther 2004;20:783-92.

21 Domenech E, Hinojosa J, Esteve-Comas M, Gomollón F, Herrera JM, Bastida G, Obrador A, Ruiz R, Saro C, Gassull MA, for the Spanish Group for the Study of Crohn's Disease and Ulcerative Colitis (GETECCU): Granulocytapheresis in steroid-dependent inflammatory bowel disease: a prospective, open, pilot study. Aliment Pharmacol Ther 2004;20: 1347-52.
22 Suzuki Y, Yoshimura N, Saito Y, et al: A retrospective search for predictors of clinical response to selective granulocyte and monocyte apheresis in patients with ulcerative colitis. Dig Dis Sci 2006;51 (11):2031-8.

23 Saniabadi AR, Hanai H, Lofberg R, et al: Adacolumn, an adsorptive carrier based granulocyte and monocyte apheresis device for the treatment of inflammatory and refractory diseases associated with leukocytes. Ther Apher Dial 2003;7:48-59.

24 Hanai H, Watanabe F, Takeuchi K, et al: Leukocyte adsorptive apheresis for the treatment of active ulcerative colitis: a prospective uncontrolled pilot study. Clin Gastroenterol Hepatol 2003;1:28-35.

25 McCarthy DA, Rampton DS, Liu Y-C: Peripheral blood neutrophils in inflammatory bowel disease: morphological evidence of in vivo activation in active disease. Clin Exp Immunol 1991;86:489-93.

26 Rugtveit J, Brandtzaeg P, Halstensen TS, et al: Increased macrophage subsets in inflammatory bowel disease: apparent recruitment from peripheral blood monocytes. Gut 1994;35:669-74.

27 Meuret G, Bitzi A, Hammer B: Macrophage turnover in Crohn's disease and ulcerative colitis. Gastroenterology 1978;74:501-3.

28 Brannigan AE, O'Connell PR, Hurley H: Neutrophil apoptosis is delayed in patients with inflammatory bowel disease. Shock 2000;13:361-6.

29 Cassatella MA: The production of cytokines by polymorph nuclear neutrophils. Immunol Today 1995;16:21-6.

30 Nikolaus S, Bauditz J, Gionchetti P: Increased secretion of pro-inflammatory cytokines by circulating polymorph nuclear neutrophils and regulation by interleukin-10 during intestinal inflammation. Gut 1998;42:470-6.

31 Mahida YR: The key role of macrophages in the immunopathogenesis of inflammatory bowel disease. Inflamm Bowel Dis 2000;6:21-33.

32 Hanauer SB: Inflammatory bowel disease: epidemiology, pathogenesis, and therapeutic opportunities. Inflamm Bowel Dis 2006;12:S3-S9.

33 Allison MC, Dhillon AP, Lewis WG, et al (eds): Inflammatory Bowel Disease. London, Mosby 1998, pp 15-95.

34 Tibble JA, Sigthorsson G, Bridger D, et al: Surrogate markers of intestinal inflammation are predictive of relapse in patients with inflammatory bowel disease. Gastroenterology 2000;119:15-22. 
35 Limburg P, David M, Ahlquist A, Sandborn WJ: Faecal calprotectin levels predict colorectal inflammation among patients with chronic diarrhoea referred for colonoscopy. Am J Gastroenterol 2000; 95:2831-7.

-36 Yokoyama Y, Fukunaga K, Fukuda Y, et al: Demonstration of low CD25High+CD4+ and high CD28-CD4+ T-cell subsets in patients with ulcerative colitis: modified by selective leucocytapheresis. Dig Dis Sci 2007:52(10):2725-31.

37 Kashiwagi N, Sugimura K, Koiwai H, Saniabadi A: Immunomodulatory effects of granulocyte and monocyte adsorption apheresis as a treatment for patients with ulcerative colitis. Dig Dis Sci 2002;6: 1334-41.

38 Andoh A, Tsujikawa T, Inatomi O, et al: Suppression of inflammatory cytokines secretion by granulocyte/ monocytes adsorptive apheresis in active ulcerative colitis. Ther Apher Dial 2005;2:123-7.

39 Shimoyama T, Sawada K, Hiwatashi N, et al: Safety and efficacy of granulocytes and monocyte adsorption apheresis in patients with active ulcerative colitis: a multicenter study. J Clin Apher 2001;16:1-9.

40 Hiraishi K, Takeda Y, Shiobara N: Studies on the mechanisms of leukocyte adhesion to cellulose acetate beads: an in vitro model to assess the efficacy of cellulose acetate carrier-based granulocyte and monocyte adsorptive apheresis. Therap Apher Dial 2003;7:334-40.

41 Matts SGF: The value of rectal biopsy in the diagnosis of ulcerative colitis. Q J Med 1961;120: 393-407.

42 Fukunaga K, Fukuda Y, Sawada K, et al: Poorly controlled ulcerative colitis treated by colectomy during remission induced by extracorporeal leukocyte removal therapy. J Gastroenterol 2003;38: 684-9.

43 Lichtiger S, Present DH, Kornbluth A: Cyclosporine in severe ulcerative colitis refractory to steroid therapy. N Engl J Med 1994;330:1841-5. 